\title{
SIMPLIFYING DATABASE NORMALIZATION WITHIN A Visual INTERACTIVE Simulation MODEL
}

\author{
Dr. Belsam Attallah \\ Senior Fellow, Higher Education Academy (HEA), UK \\ Member, British Computer Society (BCS), UK \\ Assistant Professor, Department of Computer Information Science, Higher Colleges of \\ Technology (HCT), United Arabs Emirates
}

\begin{abstract}
Although many researchers focused on investigating the challenges of database normalization, and suggested recommendations on easing these challenges, this process remained an area of concern to database designers, developers, and learners. This paper investigated these challenges and involved Higher Education in Computer Science students learning database normalization, as they could well represent beginning database designers/developers, who would struggle in effectively normalize their database design due to the complexity of this theoretical process, which has no similar real-life representation. The paper focused on the advantages of interactive visualization techniques to simplify database normalization, and recommended virtual world technologies, such as 'Second Life', as an effective platform to achieve this visualization via a simulated model of a relational database system. The simulation technique presented in this paper is novel, and is supported by extensive evidence on its advantages to achieve an illustration of the 'Normal Forms' and the need for them.
\end{abstract}

\section{KEYWORDS}

Database Normalization; Database Normalisation; Normal Forms; Visualization; Visualisation; Visual; Virtual Worlds; Second Life; Virtualization; Virtualisation.

\section{GOALS AND METHODS}

The goals of this research are to assemble literature related to the difficulties faced in the understanding and implementation of database normalization by beginning designers and developers, to investigate the advantages of visualization and interactivity to this process and to recommend an effective simulation technique to achieve this visualization. For this, Higher Education in Computer Science students have been selected for this research activities.

Both quantitative and qualitative research methods were applied to achieve the outcomes of this research (questionnaires, observations and students' feedback). An intensive literature review has been carried out to document the problem formulation, and to support the research outcomes and recommendations.

\section{INTRODUCTION}

There is significant research acknowledging the level of complexity in the database normalization process, and how this issue forms a cause of concern not only for beginning database designers and developers, but also for Higher Education (HE) students studying this process in their university studies. To this regard, Folorunso et al. (2010, p. 25) confirmed that "relational data model theory (normalization) tends to be complex for the average designers". Research also DOI : $10.5121 / \mathrm{ijdms.2017.9304}$ 
revealed that the difficulty involved in the understanding and implementation of this process caused students' poor achievement in their HE Computer Science database modules. Gaikwad et al. (2017) highlighted that database normalization is a complex theoretical process that is hard to understand by learners, which does not facilitate encouraging people to lean it.

This paper focuses on identifying the challenges faced in the analysis, understanding and learning of database normalization process and its 'Normal Forms', and provides recommendations on the techniques and platforms needed to overcome these challenges. Visualization, simulation and interactivity were the aspects explored and recommended by this paper to facilitate and enhance the comprehension and interpretation of this process in order to support novice database designers and learners.

\section{Difficulties Of Database Normalization}

\subsection{WHAT IS 'DATABASE NORMALIZATION'}

According to Caplice et al. (2017) database normalization is a fundamental component of database management related to organizing data elements into relational tables in order to improve data integrity and reduce data redundancy. Database normalization prevents data inconsistencies, wasted storage, incorrect and outdated data.

Tutorialspoint (2017) clarified that the database normalization process ensures an efficient organization of data in database tables, which results in guaranteeing that data dependencies make sense, and also reducing the space occupied by the database via eliminating redundant data. The source added that this process is divided into three 'Normal Forms', each of which represent the guidelines of how the database structure is designed/organized at that particular level. It is possible, however, to go beyond the $3^{\text {rd }}$ Normal Form to the $4^{\text {th }}, 5^{\text {th }} \ldots$ etc. in large and complicated databases, yet, research showed that normalizing a database to the $3^{\text {rd }}$ Normal Form is sufficient.

Demba (2013) defined database normalization as the process needed in a relational database, which organizes data and minimizes its redundancy. The source clarified that the normalization process involves classifying the data into two or more tables, where relationships are defined to connect the tables so that any modifications to the data (additions, deletions, alterations) in one table will then disseminate to the rest of the tables within the database via the defined relationship(s). Demba (2013, p. 39) added that the database normalization concept and its 'Normal Forms' were originally invented by Edgar Codd, the inventor of the relational model, and that the 'Normal Forms' provide "the criteria for determining a table's degree of vulnerability to logical inconsistencies and anomalies. The higher the normal form applicable to a table, the less vulnerable it is".

Looking more into the background of database normalization, Malaika et al. (2012, p. 3) clarified that database normalization was first introduced in 1970s. They defined database normalization as "a methodology that minimizes data duplication to safeguard databases against logical and structural problems, such as data anomalies". They added that the normalization process ensures that any data element, such as name, age, address, product information...etc. appears only one time on the disk in order to save storage space and avoid data inconsistencies. 
International Journal of Database Management Systems ( IJDMS ) Vol.9, No.3, June 2017

\subsection{Researchers ACKnowledging The Difficulties Faced By Database DESIGNERS AND LEARNERS In UNDERSTANDING AND IMPLEMENTING DATABASE NORMALIZATION}

Research revealed that a number of tools were designed to support the understanding, learning and implentation of database normalization, regardless of which, the subject remained an issue of concern when aiming to achieve an effectively normalized database system. Wingenious (2005) confirmed that besides being a complex subject to learn, database normalization is a critical part of an effective database design, which is vital in guaranteeing data integrity and eliminating data redundancy in a database system. Alappanavar et al. (2013) supported this by explaining how difficult it is to motivate students to learn database normalization because they consider this subject to be dry and purely theory-based. They clarified that the more the database grows, the more difficult it becomes to manually handle the normalization process.

Tutani (2015, p. 218) highlighted that it is challenging to teach conceptual database design to novice learners, in addition to being challenging for educators as well. The author added that "normalization of relations, from first to higher normal forms is often a problematic concept for students to understand". Due to the strong mathematical jargons in the normalization process, the author added, even HE learners at higher levels of databases' programmes still find it challenging to conceptualize the process, as the difficulty persists at all levels.

In the same regard, Folorunso et al. (2010, p. 26) confirmed that students in tertiary academic institutions find it difficult to learn the subject of database design theory, and in particular, the database normalization process, as "the normalization algorithms often require extensive relational algebraic backgrounds that most computer science students lack". They also clarified that researchers and educators in the databases field emphasized the complexity of this process throughout the years since the introduction of Codd's (1970) seminal work on 'Database Normal Forms'. They highlighted that the database normalization process is not only a cause of poor designer performance, but it is also a challenging subject to teach.

In a study carried out on the engineering students of SzentIstván University, Hungary, Czenky (2014) defined the process of database normalization as being a principal database design method. However, she added, the students found the understanding and application of this method problematic and challenging. She explained the outcomes of the survey carried out for this in the university in 2008, where 54 students participated. The survey revealed that $69.6 \%$ of students found database normalization as the most difficult subject. She also compared the outcomes of this survey to a similar one carried out at the University of Ulster, Northern Ireland, where the students were given the opportunity to select from (very difficult, difficult, easy and very easy) options. The summation of 'very difficult' and 'difficult' choices for the database normalization subject was $84.6 \%$ of the students.

The difficulty of the theory concepts in the database normalization process was also explained by Kung et al. (2006), who suggested designing a web-based tool to provide the learners with an interactive hands-on experience to improve the understanding and implementation of this process. They clarified the difficulty they faced in motivating their students, who considered it such a dry and theoretical subject, to learn the process. They added that the results of the survey carried out, in a junior level 'Systems Analysis and Design' course, to record students' feedback, confirmed that the learners generally viewed the web-based tool more positively than the database normalization techniques in the textbook.

Demba (2013, p. 39) highlighted that designers find the database normalization process difficult and cumbersome, as violating any of the three 'Normal Forms' criteria of normalization exposes 
the database system to problems, such as redundant data and inconsistent dependencies. The source added that designers must also be aware of "partial and transitive dependencies on all candidate keys and not just the primary key. This can make the process of normalization more complex".

\subsection{ReSEARCHERS REALIZING The NEEd For Visualization To AID In SimPLifying The Process Of Database Normalization}

A number of researchers acknowledged the need for visualization in this field. Gaikwad et al. (2017, p. 1324) stated that "Some toolsets such as NORMIT, Web based Normalization Tool, etc., are specifically designed for learning/ teaching/ understanding the process of normalization, since the process is difficult to understand and theoretical". However, Patwardhan et al. (2010) clarified that web-based normalization tools, such as NORMIT, do not provide visual aid for the normalization process. Gaikwad et al. (2017) added that other available automatic normalization tools, such as Formalizer, JMathNorm...etc., perform database normalization on the relational level only, not on the conceptual level, which does not facilitate the full understanding and learning of the normalization process.

Folorunso et al. (2010) highlighted the need for visualization to support the understanding and implementation of database normalization process. They explained their adopted visualization method, which was designed through a computer program built using $\mathrm{C \#}$ programming language, in which learners enter the database field titles and their properties in textboxes and press the buttons available in the user-interface to produce the normalization form associated with each stage. However, using this program did not enable the students to immerse in the process and generate the normalization forms themselves. Although this technique demonstrated simple/basic visualization, Folorunso et al. (2010) still confirmed that this visualization idea caused the students to view this experience more positively than the textbook technique.

Ochs (2010) explained a more advanced visualization attempt in this field in USA, which was carried out in virtual worlds. However, this attempt was to use the 3D virtual world environment to teach an introductory database course to Master of Science in Database Technology students in order to reduce the time needed to learn database concepts. The 3D model built for this purpose handled preliminary database aspects to enable students to create a Microsoft Access inventory database. It did not particularly focus on visualizing the challenging theory concepts of database normalization. The virtual model was a purposely furnished house in the virtual world of 'Second Life', with descriptions and notecards attached to different furniture objects to provide the students with information on the fields required per database table. The inventory database for this house was rather simple and did not require a major normalization process. Ochs (2010) clarified that students' feedback showed that $75 \%$ of them agreed that this model reduced the time needed to learn these concepts.

According to Taofiki et al. (2011, p. 4), a web-based learning environment was designed by Nikolay Georgiev in 2007 called LDBN (Learn Database Normalization) by using javascript and Ajax. In this platform, students are able to test their information on the relational database normalization subject online via choosing an assignment submitted by the lecturer and try to solve it. However, "LDBN cannot handle multi-valued dependencies (MVD) and thus cannot handle higher normal forms such as $4 N F$ and above". Taofiki et al. (2011) highlighted another attempt to visualize database normalization in 2009, which was carried out by Murray \&Guimaraes. This attempt was in the form of 'Animated Courseware Support for Teaching Database Design', which provided supporting material for teaching database design concepts; however, it did not actually aim at visualizing the process itself to facilitate its understanding and 
learning. Taofiki et al. (2011) explained their own developed visualization tool, from 2008-2010, which was also used as an assessment instrument to support the teaching of database decomposition techniques. This was a software tool, with a user interface that includes a number of textboxes for students to fill-in with field names/types of a database, which again did not focus on the visualization of the actual normalization process.

In the above explained literature review, there was no real visualization-focused tool that adopted simulation and fostered interactivity to simplify the process of database normalization and facilitate its understanding and implementation. This paper focuses on the employment of an emerging computing technology to achieve an effective visualization of the database normalization process, which presents a visual, user-friendly, interactive, collaborative, experimental and engaging interface, in order to facilitate grasping the complex theoretical concepts of database normalization.

\section{The Application Of Virtual World Technologies To Visualize The Database Normalization Process}

This research focused on a different visualization technique to the ones mentioned in the earlier sections. It involved the application of virtual world technologies to visualize complex theory concepts of database normalization in order to support novice database designers and learners. The research activities involved HE in Computer Science students in England, UK.

Purposely-built visualization scenarios were designed in the virtual world of 'Second Life' to support the analysis and interpretation of challenging database normalization concepts as part of the databases modules of HE in Computer Science, Year-1. These visualization scenarios were programmed by the researcher using the programming language embedded within Second Life, called 'Linden Labs Scripting Language (LSL)'. The visualization and simulation of database 'Normal Forms', and the interactivity achieved within the virtual platform, facilitated the understanding and effective implementation of the database normalization process to a high level.

In order to get an accurate comparison between the outcomes of understanding this process using the visualized technique (in virtual worlds) and the non-visualized technique (in the physical world), the researcher decided to divided the students into two groups, one to learn database normalization in the physical world and the other in the virtual world. These groups were then swapped to enable them to compare the advantages and limitations of learning the normalization process within each environment.

As students' grades could be used as a strong/valid indication of their learning and achievement, the following approaches were followed in order to enable an accurate judgement of students' benefits from visualizing the normalization process in virtual worlds, and also to avoid including irrelevant data:

1. Where one (or more) of the course assessments, included in the calculation of grades, does not involve the application of database normalization in virtual worlds, their grades were not considered.

2. Where one (or more) of the assessments, included in the calculation of grades, was carried out before using virtual worlds, their grades were also excluded.

The visualization of database normalization in virtual worlds was applied in a number of database-related modules in the selected HE programme (as explained below), and a comparison of outcomes was also carried out in order to provide further evidence on the advantages and limitations of this technique and the suggested recommendations. 
In order to visualize the complex theory concepts of the database normalization process, a clear real-life example, which requires a large database system, was chosen for the virtual scenario. This was a hospital that requires a database system to store and manage patients' diagnosis and treatment records.

Although the type of stored information on patients in a real-life hospital is obviously intuitive, the aim, when the researcher designed the virtual scenario, was to put the students, virtually, in the atmosphere of a real hospital situation. This was intended to inspire the students to investigate the certain departments (within the hospital) that need to be referred to for information on the database system in hand, and the correct approach to data exploration and gathering.

A sample hospital of two storeys was designed in Second Life. The first floor included the reception area, the pharmacy department and a couple of consultant rooms, while the second floor contained two patient wards of two different departments. There was a number of sign boards distributed within the scenario displaying information on each department, and also some metaphors of information givers, e.g. a telephone figure titled 'Call for help', which gives a notecard when clicked explaining how the patient's data is linked to that of the ward, and also a drug bottle titled 'Click for information' which provides a notecard explaining the information recorded for drugs in the pharmacy department. These explanations represented the fields required in the patient, ward and drug tables within the hospital database system. In addition, a number of 'clues' were distributed randomly within the scenario, e.g. signs and title of objects, to encourage the students to explore all the areas of the virtual hospital investigating and seeking data in order to get all the information they need to design the relational database system. Another type of information was embedded in a bookshelf titled 'Hospital Files', the books of which had individual URL links to published webpages that were purposely designed and scripted to provide further information on the relationships between the data of different tables within the database system. See Figures 3.1 and 3.2 below.

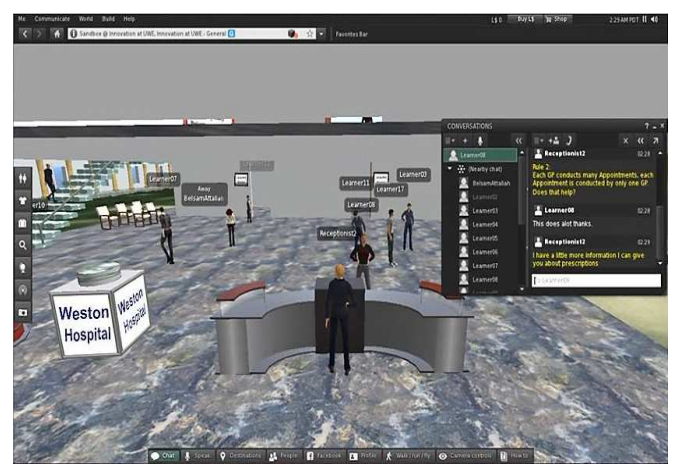

Figure 3.1: The Database Normalization virtual scenario (Weston Hospital) 


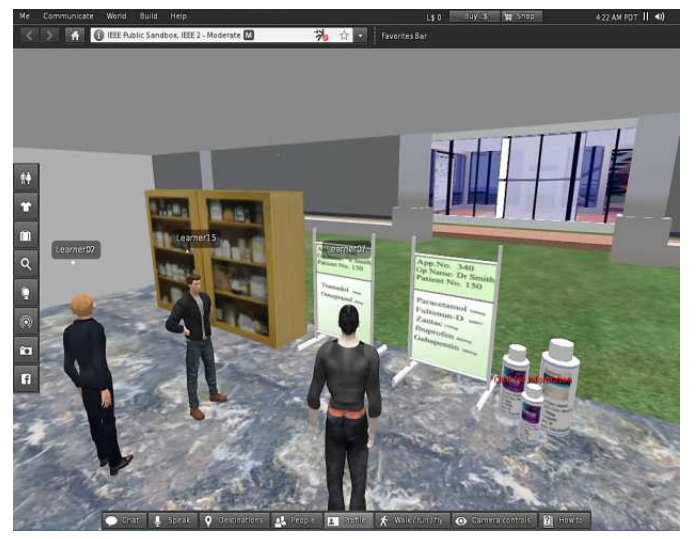

Figure 3.2: The Database Normalization virtual scenario (Pharmacy Department)

This virtual scenario was designed to mirror the actual procedure that the database designer need to follow when visiting a real hospital, i.e. exploring all the departments involved, investigating sources of information, e.g. hospital records and staff members, and writing comments based on their own observation of the hospital procedures and how patients' data is processed.

Following collecting all the information needed for the hospital database example, the students were provided with virtual sculptures of the database components so that they use them to design a 3D visual representation of the database system. These sculptures represented the fields of tables within the database, which students were required to use in order to normalize the individual tables starting from the ' 1 st $N$ ormal Form' and proceeding to the ' 3 rd Normal Form'. See Figures 3.3 and 3.4 below.

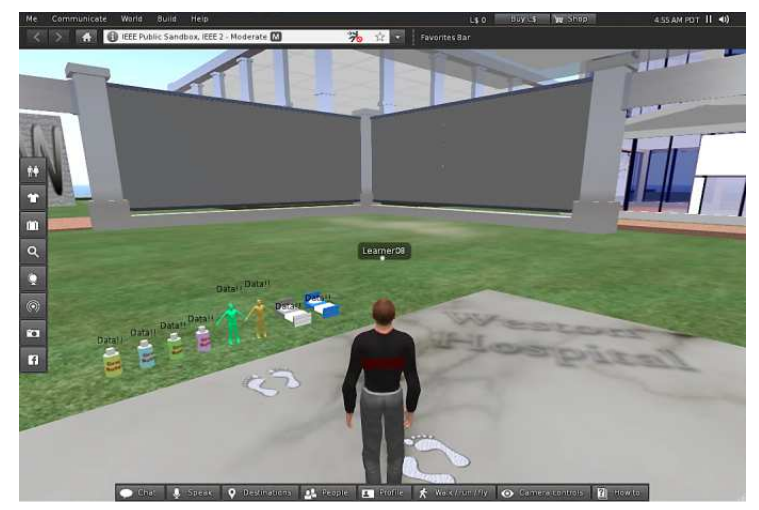

Figure 3.3: The Database Normalization virtual scenario (sculptures representing table fields) 


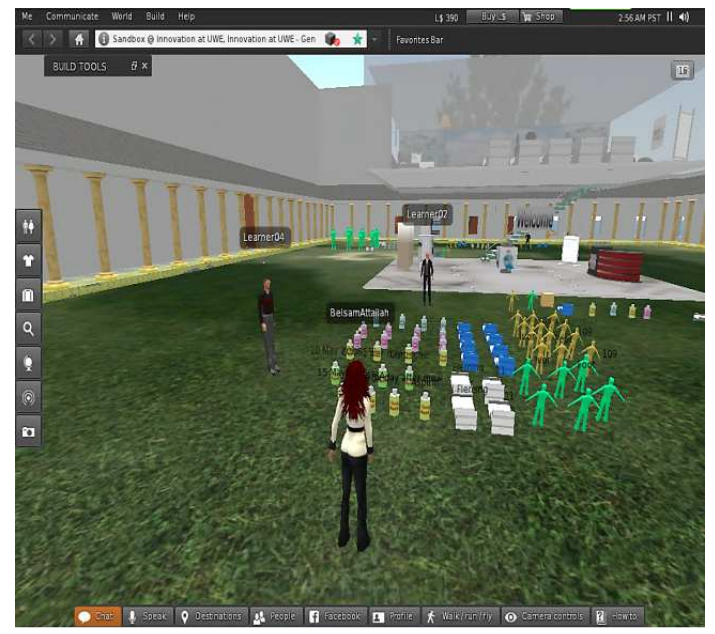

Figure 3.4: The Database Normalization virtual scenario (students normalizing database tables visually)

The students were required to move the sculptures (the data fields within the database) and rearrange them in rows (patients' records) and columns (table fields) and keep adding, removing, restructuring and separating sculptures throughout the Normal Forms. The observations by the researcher confirmed that the students found the scenario extremely useful in visualizing why the 'Three Normal Forms' are necessary to improve data integrity within the tables and to eliminate unnecessary duplication(s) and repetition within the data. This visualization enabled the understanding of why the normalization process is vital for the success of a relational database system and the critical need to an effective and logical design of its data tables. As students were seeing the immediate outcomes of their structuring process of the database tables and fields, they were able to see the impacts of certain aspects, e.g. data duplication and repetition, and consequently were able to do the correct changes to the design of their tables to eliminate the problems and successfully achieve a fully normalized database.

These observations by the researcher were then approved by the outcomes of the questionnaire completed by the students following the virtual exercise regarding the enhancement to their understanding, learning and implementation of this complex theory process.

As indicated earlier within this section, the HE students were divided into two groups, where both groups received a basic introduction to the normalization process via talks by the lecturer and some explanations on the whiteboard. This was then followed by delivering the whole normalization exercise to the first group using the traditional method (in the physical world), and the whole exercise to the second group using the visualized scenario (in the virtual world). The two groups were then swapped to have the same exercise (in the following session) using the other method, to enable them to have an effective comparison between their achievement within each platform, and also to reflect more on the advantages and limitations of each environment, e.g. engagement, collaboration, interactivity and affective quality.

The outcomes of the questionnaire distributed to Year-1 (Section-1) students to capture and record their feedback are explained below. The results of the questionnaire showed that $53 \%$ of the students considered database normalization as a difficult subject, however, only $49 \%$ of them agreed that exercising the process in the virtual world environment enhanced their learning and understanding of its complex theory concepts, while $57 \%$ of them agreed to enhanced affective quality and $57 \%$ found this learning process more engaging (see Figure 3.5 below). 


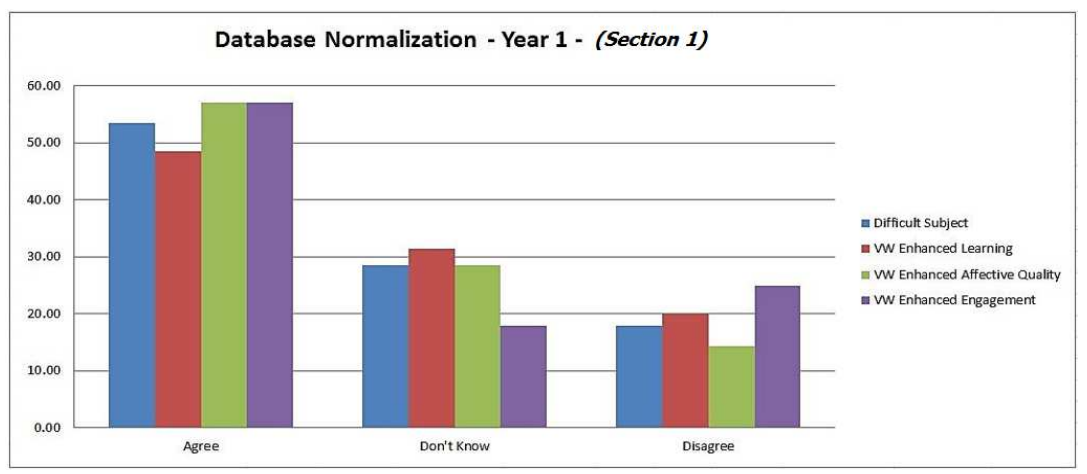

Figure 3.5: Statistics of Database Normalization, Year-1 (Section-1)

It is worth mentioning here that the researcher carried out some vital improvements to the database virtual scenario following the first virtual exercise based on students' feedback and discussions following the exercise. This included providing more information on the types of tables needed for the database and the fields within each of them. This facilitated the understanding of the 'Relational Database' concept, which is fundamental in the database normalization process. This resulted in more positive outcomes for the normalization exercises of the subsequent Year-1 sections.

Moving to Year-1 (Section-2), and following the improvements made on the virtual scenario, a different approach to recording students' feedback was adopted compared to that in Year-1 (Section-1). Students' feedback was captured and recorded before and after the application of virtual world technologies to visualize the database normalization process.

The questionnaire produced the following outcomes (see Figure 3.6 below):

- Slightly more than half of the students who originally agreed that this subject is deemed difficult changed their minds following practicing in the virtual world scenario.

- Twenty-two percent more students agreed that effective understanding and implementation of the normalization process took place in virtual worlds, which facilitated their learning of its complex theory concepts.

- Sixteen percent more students agreed that affective quality was enhanced.

- Twenty-three percent more students found virtual worlds more engaging when working on the normalization process.

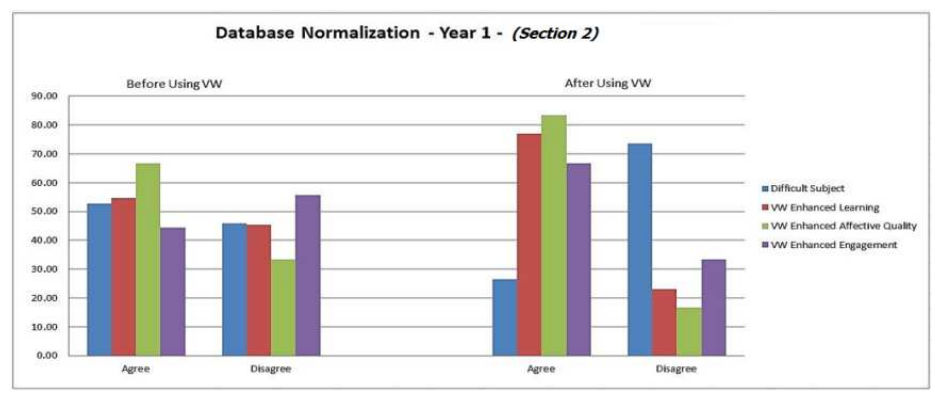

Figure 3.6: Statistics of Database Normalization, Year-1 (Section-2) 
For a more in-depth comparison, and by looking again into the analysis achieved above for Year1 (Section-1), the researcher carried out further investigation to answer the questions in Table 3.1 below:

\begin{tabular}{|l|l|}
\hline Question & Percentage \\
\hline $\begin{array}{l}\text { 1. What is the percentage of HE students who found the database normalization } \\
\text { process difficult but their understanding and implementation of the process } \\
\text { were enhanced following the visualization achieved in virtual worlds? }\end{array}$ & $50 \%$ \\
\hline $\begin{array}{l}\text { 2. What is the percentage of HE students who found the database normalization } \\
\text { process difficult but their affective quality was enhanced following practicing } \\
\text { in virtual worlds? }\end{array}$ & $67 \%$ \\
\hline $\begin{array}{l}\text { 3. What is the percentage of HE students who found the database normalization } \\
\text { process difficult but their engagement in the process was enhanced following } \\
\text { practicing in virtual worlds? }\end{array}$ & $83 \%$ \\
\hline $\begin{array}{l}\text { 4. What is the percentage of HE students who found the database normalization } \\
\text { process difficult but their understanding and implementation of the process } \\
\text { were not enhanced following the visualization achieved in virtual worlds? }\end{array}$ & $50 \%$ \\
\hline $\begin{array}{l}\text { 5. What is the percentage of HE students who did not find the database } \\
\text { normalization process difficult but their understanding and implementation of } \\
\text { the process were not enhanced following the visualization achieved in virtual } \\
\text { worlds? }\end{array}$ & $0 \%$ \\
\hline
\end{tabular}

Table 3.1: Data correlation of the Database Normalization exercises, Year-1 (Section-1) Moving to Year-1 (Section-2), the five questions were rephrased in order to reflect the additional element of before and after the application of virtual world technologies that was added to the questionnaire following the improvements on the original virtual scenario. The outcomes were as in Table 3.2 below:

\begin{tabular}{|c|c|}
\hline Question & Percentage \\
\hline $\begin{array}{l}\text { 1. What is the percentage of HE students who found the database normalization } \\
\text { process difficult (before the application of virtual worlds), but their } \\
\text { understanding and implementation of the nomalization process were enhanced } \\
\text { (after the visualization achieved in virtual worlds)? }\end{array}$ & $100 \%$ \\
\hline $\begin{array}{l}\text { 2. What is the percentage of HE students who found the database normalization } \\
\text { process difficult (before the application of virtual worlds), but their affective } \\
\text { quality was enhanced (after the visualization achieved in virtual worlds)? }\end{array}$ & $100 \%$ \\
\hline $\begin{array}{l}\text { 3. What is the percentage of HE students who found the database normalization } \\
\text { process difficult (before the application of virtual worlds), but their engagement } \\
\text { in the process was enhanced (after the visualization achieved in virtual worlds)? }\end{array}$ & $70 \%$ \\
\hline $\begin{array}{l}\text { 4. What is the percentage of HE students who found the database normalization } \\
\text { process difficult (before the application of virtual worlds), but their } \\
\text { understanding and implementation of the process were not enhanced (after the } \\
\text { visualization achieved in virtual worlds)? }\end{array}$ & $0 \%$ \\
\hline $\begin{array}{l}\text { 5. What is the percentage of HE students who did not find the database } \\
\text { normalization process difficult (before the application of virtual worlds), but their } \\
\text { understanding and implementation of the process were enhanced (after the } \\
\text { visualization achieved in virtual worlds)? }\end{array}$ & $67 \%$ \\
\hline
\end{tabular}


Table 3.2: Data correlation of the Database Normalization exercises, Year-1 (Section-2)

The above strong percentages were confirmed by the high module grades for Section-2, as shown below (see table 3.3):

\begin{tabular}{|l|l|l|l|}
\hline Year \& Section & $\begin{array}{l}\text { Year-1 } \\
\text { (a previous section } \\
\text { without visualizing in } \\
\text { virtual worlds) }\end{array}$ & Year-1 (Section-1) & Year-1 (Section-2) \\
\hline $\begin{array}{l}\text { Database Nomalization } \\
\text { Databases Module }\end{array}$ & $\begin{array}{l}97.16 \\
\text { StDev. }=7.7\end{array}$ & $\begin{array}{l}99.12 \\
\text { StDev. }=5.4\end{array}$ & 106.58 \\
\hline
\end{tabular}

Note: Grade $=($ Average mark of the module / Average mark of all Computing modules $* 100)$

Table 3.3: Grades of the Databases module, Year-1

The Web Technologies module in Year-1 was another strong evidence on the advantages of visualizing the database normalization process in virtual worlds. Within this module, students are expected to program their website and also apply their understanding of database normalization in their design of the online MySQL database required for their website. The database normalization skills needed in this module were applied towards the end of the academic year in the web practical project, which is the final assessment in this module. The grades of this practical assessment clearly showed the development in students' understanding and learning of the database normalization complex theory concepts, which were re-applied as part of the web project. The grades were as in Table 3.4 below:

\begin{tabular}{|l|l|l|l|}
\hline Year \& Section & $\begin{array}{l}\text { Year-1 } \\
\text { (a previous section } \\
\text { without visualizing in } \\
\text { virtual worlds) }\end{array}$ & Year-1 (Section-1) & Year-1 (Section-2) \\
\hline $\begin{array}{l}\text { Web Technologies, Yesr-1 } \\
\text { (Practical Assessment 2 - web } \\
\text { project) }\end{array}$ & 99.69 & 109.66 & 115.51 \\
\hline
\end{tabular}

Note: Grade $=($ Average mark of the module / Average mark of all Computing modules $* 100)$ Table 3.4: Grades of the Web Technologies module, Year-1

In addition to the above, the feedback received by the other HE lecturer delivering the theory of database normalization using the traditional way, reinforced the fact that students' understanding and learning of the complex theory concepts of this process were noticeably, if not greatly, enhanced following the visualization achieved in virtual worlds. This lecturer managed to observe students' performance and attitude before and after the application of virtual worlds. She said: “...The virtual scenarios had a positive impact on areas of particular difficulty... The visualization reinforced some of the more challenging concepts... Scenario ideas are a useful addition to learning aids... The virtual world is a powerful tool that can be used to make the constructs more memorable... Initial suspicion and some reluctance were gradually replaced by interest and then acknowledgement of the value of this addition to their curriculum". In reinforcing the advantages of visualization, she added that as many people are visual learners, "the virtual scenario helped them to understand the rules more fully and see the outcome of the reduction of duplication that normalization brings... Students felt more able to attempt the normalization process... It made a positive impact, and students were interested to see the change 
in the objects following each stage of the normalization process... Objects and situations are always much more interesting than raw data".

\section{CONCLUSION}

Understanding the complex theory concepts of database normalization forms a cause of concern to a large number of database designers and developers, in addition to people studying this field at the HE level. Research revealed that these concerns are within the main reasons behind achieving poorly or failing the modules that include database normalization concepts.

Research also showed a number of software and online tools, which aimed at illustrating the process of database normalization for novice designers and learners; however, the majority of them focused on automating the process rather than visualizing and simplifying it to facilitate its understanding and effective implementation, which did not generate the degree of support needed for this complex theory process.

This research demonstrated that there are strong indications of the advantages of visualizing database normalization in virtual worlds, as this platform offers great benefits such as interactivity, collaboration and experimentation, which are ideal for understanding and learning the normalization process. This did not only cover enhancements to people's understanding, but also increased their engagement in the process, enhanced affective quality and improved their achievement of effectively normalized database systems.

\section{REFERENCES}

[1] Alappanavar, P.B., Grover, R., Hunjan, S., Patil, D. and Girnar, Y. (2013) Automating the normalization process for relational database model. International Journal of Engineering Research and Applications (IJERA). ISSN: 2248-9622, January -February 2013. 3 (1), pp.1826-1831.

[2] Caplice, C., Ponce, E. (2017) MicroMasters in Supply Chain Management. V1 Spring 2017. CTL.SC4x - Technology and Systems. MIT Center for Transportation \& Logistics. Cambridge, MA 02142 USA.

[3] Czenky, M. (2014) The Efficiency Examination of Teaching of Different Normalization Methods. Department of Informatics, SzentIstván University, Gödöllö, Hungary. International Journal of Database Management Systems (IJDMS). 6(2), pp. 1-18.

[4] Demba, M. (2013) Algorithm for Relational Database Normalization up to 3nf. International Journal of Database Management Systems (IJDMS). 5(3), pp. 39-51.

[5] Folorunso, O., Akinwale, A.T. (2010) Developing visualization support system for teaching/learning database normalization. Emeraldinsight, Campus-Wide Information Systems Journal, 27 (1), pp.2539. Emerald Group Publishing Limited, UK.

[6] Gaikwad, A.S., Kadri, F.A., Khandagle, S.S., Tava, N.I. (2017) Review on Automation Tool for ERD Normalization. International Research Journal of Engineering and Technology (IRJET) [Online]. 4 (2), pp. 1323-1325. [Accessed 07 May 2017]. Available from: https://www.irjet.net/archives/V4/i2/IRJET-V4I2259.pdf

[7] Kung, H. J., Tung, H. L. (2006) A web-based tool to enhance teaching/learning database normalization. Proceedings of the 2006 Southern Association for Information Systems Conference, pages 251-258. Georgia Southern University \& Troy University, USA.

[8] Malaika, S., Nicola, M. (2012) Data Normalization Reconsidered. Part 1. DeveloperWorks, IBM [Online]. $\quad$ [Accessed 20 May 2017]. Available from: https://www.ibm.com/developerworks/data/library/techarticle/dm-1112normalization/ 
[9] Ochs, J. L. (2010) Database concepts in a virtual world. Thesis, Department of Information Systems, School of Computer \& Information Sciences, Regis University, USA. In partial fulfillment of the requirements of Master of Science in Database Technology.

[10] Patwardhan, M.S., Dhabe, P.S., Deshpande, A.A., Londhe, S.G., Dhore, M. L. and Abhyankar, H. K. (2010) Diagrammatic approach for complete automation of relational database normalization at conceptual level. International Journal of Database Management Systems (IJDMS). 2 (4), pp. 132151.

[11] Taofiki, A.A., Tale, A.O. (2011) A Visualization Tool for Teaching and Learning Database Decomposition System. Department of Computer Science, University of Agriculture, Abeokuta, Nigeria. Journal of Information and Computing Science. 7(1), pp. 03-10.

[12] Tutani, D. (2015) Teaching Database Normalization at first year University Level: A Simplified Approach. Department of Business Information Systems, University of Venda, South Africa. The 2015 International Academic Research Conference, 3-6 August, University of London, UK. ICBTS Copyright.

[13] Tutorialspoint (2017) SQL Structured Query Language. Simply Easy Learning [Online]. [Accessed 07 May 2017]. Available from: http://www.tutorialspoint.com/sql/sql_tutorial.pdf

[14] Wingenious (2005) Database Architecture. 4th edition. Library3.org [Online]. [Accessed 15 December 2016]. Available from: http://www.wingenious.com/database.pdf 